\title{
Necessity of structured exercise training program and its feasibility on physical capacity and health status with post COVID-19 syndrome patients
}

Ruhi kumbhare ( ruhikumbhare2009@gmail.com )

Datta Meghe Institute of Medical Science's https://orcid.org/0000-0002-7803-736X

Dr. VISHNU VARDHAN ( $\nabla$ vishnudiwakarpt@gmail.com )

Datta Meghe Institute of Medical Science's

\section{Method Article}

Keywords: coronavirus disease, pulmonary Rehabilitation syndrome, Pulmonary Rehabilitation exercise Protocol , Post COVID Symptoms, Exercise Prescription Protocol

Posted Date: June 14th, 2021

DOl: https://doi.org/10.21203/rs.3.pex-1541/v1

License: (c) (i) This work is licensed under a Creative Commons Attribution 4.0 International License.

Read Full License 


\section{Abstract}

Corona virus disease 2019 (COVID-19) is widespread and has become a global public health emergency. The disease's outbreak was recently announced by the World Health Organization. Pulmonary Rehabilitation has been shown to improve patients' quality of life, function, and well-being with Chronic Respiratory Diseases Recently there have been various guidelines and consensus available for Pulmonary Rehabilitation in Post - COVID patients who have been discharged from other respiratory conditions. There is a limited number of literatures on the effect of exercise program on physical fitness and health status of patients with Post COVID 19 syndrome. The current study therefore uses the available evidence in COVID 19 patients to modify an exercise program and apply it to patients with COVID syndrome. The Central Trial Registry of India (CTRI) registration number for this trial is REF/2021/06/044205.

\section{Introduction}

Coronavirus Disease 2019 (COVID-19) is widespread and has risen to the level of a global public health emergency. The World Health Organization recently announced the disease's outbreak.

The so-called "Post-COVID Syndrome" refers to symptoms that persist after treatment and may be related to residual inflammation (seizures), physical injuries, side effects from the hospital or chronic shortness of breath (post-intensive care syndrome), isolation or impact on health conditions already existing.

The definition of pulmonary rehabilitation, as derived from the American Thoracic Society/European Respiratory Society, is a comprehensive intervention based on comprehensive patient evaluation followed by patient-centered therapies that include, but are not limited to, training, education, and behavioral changes designed to improve respiratory status.

The goal of rehabilitation in COVID-19 patients is to alleviate dyspnea symptoms, eliminate anxiety, lose weight, reduce disability, maintain function, and improve health. Each patient should have a plan for lung rehabilitation..

When possible and safe, pulmonary rehabilitation should be considered during dynamic administration of COVID-19, and may include nutrition, ventilation, standing, clearing process, oxygen supplementation, breathing exercises, stretching, and strengthening exercises. Given the possibility of long-term disability, lung rehabilitation following hospitalization is recommended..

An important part of rehabilitation is restoring strength and independence in exercise training. Cardio breathing exercises are related to making a large, strong, balanced muscle group go to great exercise for a long time.

The goal of this study is to look into the effect of a 6-week Physical Rehabilitation programme on Post COVID syndrome patients' physical fitness (cardiovascular fitness) and health-related quality of life. 


\section{Reagents}

\section{Equipment}

dumbells, sphygnomanometer, pulse oximeter,therabands,weight cuffs, treadmills

\section{Procedure}

Initial contact with patients will be made by telephone interview or by direct appointment from Respiratory OPD. Patients will be evaluated according to the inclusion and exclusion criteria. Inform consent Patients will be taught to wear comfortable clothes, walking shoes and masks. There is a 2-team supervised group and an unsupervised group. In the supervised group is defined the certified Pulmonary Rehabilitation Protocol

GROUP A :- Supervised group consists of Aerobic training Exercises, resistance exercise ,balance training exercise, Respiratory training exercises. The Exercise Training program is prescribed on the basis of FITT (Frequency, Intensity, Type, Time) principle. The exercise training program will be started from day 2 data will be calculated, statistical analysis will be calculated .The treatment period of Supervised Group will be of six weeks

GROUP B:- In Unsupervised groups same as group A Exercise Training Protocol will be given, detailed explanation of all exercises and techniques will be taught and performed by the participants on day 1 ,remaining sessions will be asked to perform at home, handouts will be given to patients can contact through video calls and telephone l.e Tele- rehabilitation in between the treatment period. Treatment will be carried out for 6 weeks in each group. Tailored exercise prescription will be given. Termination indicators to training program will be-Temperature $>38.2$ degrees, Chest pain, chest tightness, Aggravated cough , Dizziness

\section{Troubleshooting}

\section{Time Taken}

1 year

\section{Anticipated Results}

Effect of Exercise Training Program on Post COVID Patients 


\section{References}

1)Rehabilitation programs for patients with COronaVIrus Disease 2019: consensus statements of Taiwan Academy of Cardiovascular and Pulmonary Rehabilitation Yuan-Yang Cheng, Chin-Ming Chen, Wei-Chun Huang, Shang-Lin Chiang i, j, Pei-Chun Hsieh

2) Guzik TJ, Mohiddin SA, Dimarco A, Patel V, Savvatis K, Marelli Berg FM, et al. COVID-19 and the cardiovascular system: implications for risk assessment, diagnosis, and treatment options. 2020;116:1666e87.

3). Driggin E, Madhavan MV, Bikdeli B, Chuich T, Laracy J, Biondi Zoccai G, et al. Cardiovascular considerations for patients, health care workers, and health systems during the COVID-19 pandemic. J Am Coll Cardiol 2020;75:2352e71.

4). Thomas P, Baldwin C, Bissett B, Boden I, Gosselink R, Granger CL, et al. Physiotherapy management for COVID-19 in the acute hospital setting: clinical practice recommendations. J Physiotherapy 2020;66:73e82. https://doi.org/10.1016/j.jphys.2020.03.011.

5). Yang J, Zheng Y, Gou X, Pu K, Chen Z, Guo Q, et al. Prevalence of comorbidities in the novel Wuhan coronavirus (COVID-19) infection: a systematic review and meta-analysis. Int J Infect Dis 2020. https://doi.org/10.1016/j.ijid.2020.03.017.

6) Shi Y, Yu X, Zhao H, Wang H, Zhao R, Sheng J. Host susceptibility to severe COVID-19 and establishment of a host risk score: findings of 487 cases outside Wuhan. Crit Care 2020;24:108.

7). World Health Organization. Clinical management of COVID-19: interim guidance. Available from: https://www.who.int/ publications/i/item/clinical-management-of-covid-19. [Accessed 24 August 2020].

8). The American College of Sports Medicine. ACSM's guidelines for exercise testing and prescription. 10th ed. Philadelphia, PA: Lippincott Williams \& Wilkins.; 2016

9) ). World Health Organization. Home care for patients with suspected or confirmed COVID-19 and management of their contacts. Available from: https://www.who.int/publications/i/item/home-care-forpatients-with-suspected-novel

coronavirus-(ncov)-infection-presenting-with-mild-symptomsand-management-of-contacts. [Accessed 24 August 2020]

\section{Figures}




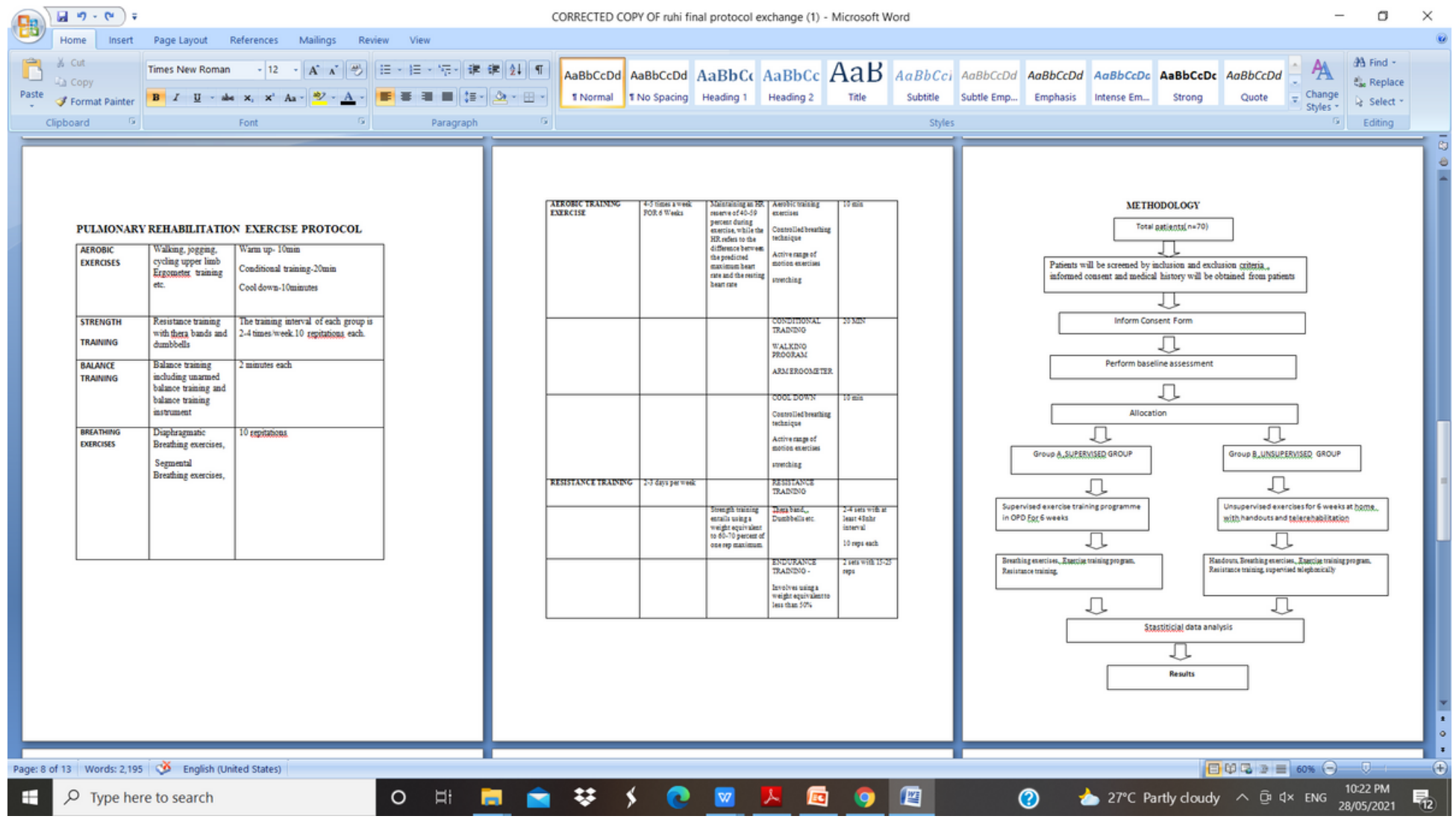

\section{Figure 1}

fig 1 - Pulmonary Rehabilitation exercise Protocol fig 2-pulmonary Rehabilitation Exercise Prescription in FITT Format fig 3- Methodology flow chart detailed description 